\title{
12.
}

\section{Two lettres of the Geometrical correspondence between M. Donkin and M. Spottiswoode.}

.... The following is partly a simplification and partly a development of a method which you once shewed me for determining two cyclie sections of a surface of the second order:

If $\theta, \mu, l, m, n, l^{\prime}, n^{\prime}, n^{\prime}, \boldsymbol{P}, \boldsymbol{Q}, \boldsymbol{R}, \boldsymbol{K}$, be any constants (I shall suppose

$$
\left.l^{2}+m^{2}+n^{2}=1, \quad l^{\prime 2}+m^{\prime 2}+n^{2}=1\right),
$$

it is clear that the surface, whose equation is

(1) $\theta\left(x^{2}+y^{2}+z^{3}\right)+\mu(l x+m y+n z)\left(l^{\prime} x+m^{\prime} y+n^{\prime} z\right)+P x+Q y+R z=K$, is cut circularly by the two systems of planes represented by

$$
\begin{gathered}
l x+m y+n z=\text { Const. } \\
l^{\prime} x+m^{\prime} y+n^{\prime} z=\text { Const. }
\end{gathered}
$$

since either of these assumptions reduces (1.) to the equation of a sphere. Now (1) will coincide with the general equation

(2) $A x^{2}+B y^{2}+C z^{2}+2 F y z+2 G z x+2 H x y+P x+Q y+R z=K$; provided we have

$$
\theta+\mu l l^{\prime}=A, \quad \mu\left(m n^{\prime}+m^{\prime} n\right)=2 \boldsymbol{F},
$$

which conditions may be written:

$$
\frac{l l^{\prime}}{A-\theta}=\frac{m m^{\prime}}{B-\theta}=\frac{n n^{\prime}}{C-\theta}=\frac{m n^{\prime}+m^{\prime} n}{2 F}=\frac{n l^{\prime}+n^{\prime} l}{2 G}=\frac{l m^{\prime}+l^{\prime \prime} m}{2 \boldsymbol{H}}=\frac{1}{\mu},
$$

from which (writing $A-\theta=A^{\prime}, B-\theta=B^{\prime}, C-\theta=C^{\prime}$ ) the following are immediately deducible:

$$
\text { (3) } \frac{m n^{\prime}-m^{\prime} n}{\sqrt{\left(F^{2}-B C\right)}}=\frac{n l^{\prime}-n^{\prime} l}{\sqrt{\left(G^{2}-C A\right)}}=\frac{l m^{\prime}-l m}{\sqrt{\left(H^{2}-A B\right)}}=\frac{2}{\mu} \text {. }
$$

We have then, if

$$
\begin{aligned}
\boldsymbol{F}^{2}-\boldsymbol{B C}=\mathrm{F}, \quad \boldsymbol{G}^{2}-\boldsymbol{C A} & =\mathrm{G}, \quad \boldsymbol{H}^{2}-\boldsymbol{A B}=\mathrm{H}: \\
m n^{\prime}+m^{\prime} n & =\frac{2}{\mu} \boldsymbol{F} \\
m n^{\prime}-m^{\prime} n & =\frac{2}{\mu} \sqrt{\mathrm{F}}
\end{aligned}
$$


whence

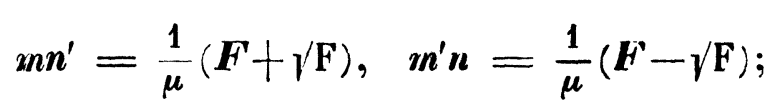

which equations, combined with

$$
m m^{\prime}=\frac{\boldsymbol{B}^{\prime}}{\mu}, \quad n n^{\prime}=\frac{\boldsymbol{C}^{\prime}}{\mu},
$$

give immediately:

$$
\begin{aligned}
& \frac{n^{\prime}}{m^{\prime}}=\frac{F+\sqrt{ } \mathrm{F}}{B^{\prime}}=\frac{C^{\prime}}{F-\sqrt{ } \mathrm{F}}, \quad \frac{m}{n}=\frac{F+\sqrt{ } \mathrm{F}}{C^{\prime}}=\frac{B^{\prime}}{F-\sqrt{ } \mathrm{F}}, \\
& \frac{l^{\prime}}{n^{\prime}}=\frac{G+\sqrt{ } \mathrm{G}}{C^{\prime}}=\frac{A^{\prime}}{G-\sqrt{ } G}, \quad \frac{n}{l}=\frac{G+\sqrt{ } G}{A^{\prime}}=\frac{C^{\prime}}{G-\sqrt{ } G}, \\
& \frac{m^{\prime}}{l^{\prime}}=\frac{H+\sqrt{ } \mathrm{H}}{A^{\prime}}=\frac{B^{\prime}}{H-\sqrt{ } \mathrm{H}}, \quad \frac{l}{m}=\frac{H+\sqrt{ } \mathrm{H}}{\boldsymbol{B}^{\prime}}=\frac{\boldsymbol{A}^{\prime}}{\boldsymbol{H}-\sqrt{ } \mathrm{H}} .
\end{aligned}
$$

Thus the six direction-cosines $l, m, n, l^{\prime}, m^{\prime}, n^{\prime}$ are completely determined as soon as $\theta$ (which is involved in $\boldsymbol{A}^{\prime}$ etc.) is known. To find an equation for determining $\theta$, we have only to substitute for $m: n, n: l, l: m$, their values in the identical equation

$$
\frac{m}{n} \cdot \frac{n}{l} \cdot \frac{l}{m}=1
$$

we find

$$
(\boldsymbol{F} \pm \sqrt{ } \mathbf{F})(\boldsymbol{G} \pm \sqrt{ } \mathbf{G})(\boldsymbol{H} \pm \sqrt{H})=\boldsymbol{A}^{\prime} \boldsymbol{B}^{\prime} \boldsymbol{C}^{\prime}
$$

But it is better to employ your original method. Reverting, namely to the equations ( 1 and 2 ), we obtain, by subtraction:

$$
\begin{gathered}
\boldsymbol{A}^{\prime} \boldsymbol{x}^{2}+\boldsymbol{B}^{\prime} y^{2}+\boldsymbol{C}^{\prime} z^{2}+2 \boldsymbol{F} y \boldsymbol{z}+2 \boldsymbol{G} \boldsymbol{z} x+2 \boldsymbol{H} x \boldsymbol{y} \\
=\mu(\boldsymbol{l} x+m y+n z)\left(l^{\prime} x+m^{\prime} y+n^{\prime} z\right),
\end{gathered}
$$

so that we have only to express the condition that the function on the lefthandside of this equation is capable of being resolved into linear factors, which, as is well known, is

$$
A^{\prime} B^{\prime} C^{\prime}-A^{\prime} F^{2}-B^{\prime} G^{2}-C^{\prime} H^{2}+2 F G H=0 .
$$

It is to be remarked, that the expressions $m n^{\prime}-m^{\prime} n$, etc. are proportional to the direction-cosines of the line of intersection of the cyclic planes; and their values being proportional (3) to $\sqrt{ }\left(\boldsymbol{F}^{2}-\boldsymbol{B}^{\prime} \boldsymbol{C}^{\prime}\right), \sqrt{ }\left(\boldsymbol{G}^{2}-\boldsymbol{C}^{\prime} \boldsymbol{A}^{\prime}\right), \sqrt{ }\left(\boldsymbol{H}^{2}-\boldsymbol{A}^{\prime} \boldsymbol{B}^{\prime}\right)$, it follows that the line in question coincides with one of the principal axes of the cone. In fact, the equation (4), multiplied by $\boldsymbol{A}^{\prime}$, may be put in the form

$$
\left(\boldsymbol{G}^{2}-\boldsymbol{C}^{\prime} \boldsymbol{A}^{\prime}\right)\left(\boldsymbol{H}^{2}-\boldsymbol{A}^{\prime} \boldsymbol{B}^{\prime}\right)=\left(\boldsymbol{G H}-\boldsymbol{F} \boldsymbol{A}^{\prime}\right)^{2}
$$


and in like manner, multiplying by $\boldsymbol{B}^{\prime}$ and $\boldsymbol{C}^{\prime}$, we set the two other forms

$$
\begin{aligned}
\left(\boldsymbol{H}^{2}-\boldsymbol{A}^{\prime} \boldsymbol{B}^{\prime}\right)\left(\boldsymbol{F}^{2}-\boldsymbol{B}^{\prime} \boldsymbol{C}^{\prime}\right) & =\left(\boldsymbol{H} \boldsymbol{F}-\boldsymbol{G} \boldsymbol{B}^{\prime}\right)^{2}, \\
\left(\boldsymbol{F}^{2}-\boldsymbol{B}^{\prime} \boldsymbol{C}^{\prime}\right)\left(\boldsymbol{G}^{2}-\boldsymbol{C}^{\prime} \boldsymbol{A}^{\prime}\right) & =\left(\boldsymbol{H} \boldsymbol{G}-\boldsymbol{H} \boldsymbol{C}^{\prime}\right)^{2},
\end{aligned}
$$

by means of which expressions it is easy to deduce the above forms for the direction-cosines of the principal axes.

But there are other important consequences to be drawn from the equation (4), when put in the three forms last written. In the first place we see that the three equations $F, G, H$, have necessarily the same sign; so that $\sqrt{ } \mathbf{F}, \sqrt{ } \mathbf{G}, \sqrt{ } \mathbf{H}$ are either all real, or all imaginary.

Next taking any one of the three forms, for example

$$
\left(\boldsymbol{G}^{2}-\boldsymbol{C}^{\prime} \boldsymbol{A}^{\prime}\right)\left(\boldsymbol{H}^{2}-\boldsymbol{A}^{\prime} \boldsymbol{B}^{\prime}\right)-\left(\boldsymbol{G}^{\prime} \boldsymbol{H}-\boldsymbol{F} \boldsymbol{A}^{\prime}\right)^{2}=\mathbf{0},
$$

let $\beta, \beta^{\prime}$ be the two values of $\theta$ which satisfy the equation $G^{2}-C^{\prime} A^{\prime}=0$ (which are necessarily real), and in like manner let $\gamma, \gamma^{\prime}$ be the two roots of $\boldsymbol{H}^{2}-\boldsymbol{A}^{\prime} \boldsymbol{B}^{\prime}=0$. Let these four quantities, arranged in order of magnitude, be called [1], [2], [3], [4]. In the above equation, which we will write thus:

$$
\mathbf{S}=\mathbf{G H}-\mathbf{A}^{2}=\mathbf{0}
$$

(which is of the fourth degree, with respect to $\theta$, having been multiplied by $\boldsymbol{A}^{\prime}$ ). Let us examine the value of $S$, while $\theta$ varies continuously from $-\infty$ to $+\infty$. We see that when $\theta=-\infty, G$ and $H$ are both negative and $\boldsymbol{S}$ is positive.

When $\theta=[1]$, we have $\boldsymbol{S}$ negative; so that there is a root of $\boldsymbol{S}$ less than [1]. But since $G$ and $H$ are negative as long as $\theta<[1]$, such a root gives imaginary values to the direction-cosines $l, m, n ; l^{\prime}, m^{\prime}, n^{\prime}$. Again no root of $\boldsymbol{S}$ can lie between [1] and [2], because it is plain that for values of $\theta$ between these limits, $G$ and $H$ have different signs. For the same reason no root can lie between [3] and [4]. When $\theta=[4]$, we have $\boldsymbol{S}$ negative, and when $\boldsymbol{\theta}=+\infty, \boldsymbol{S}$ is positive. Therefore there is one root greater than [4]; but this again gives imaginary direction-cosines, because $G$ and $H$ are both negative, when $\theta>[4]$. Of the two remaining roots, one is given by $A^{\prime}=0$, or $\theta=A$, and is extraneous to the geometrical question, having been introduced by multiplication. But it will enable us to shew that the fourth root lies between [2] and [3]. For since $\theta=[2]$ and $\theta=[3]$ both give $\boldsymbol{S}$ negative, both roots, or else neither, must lie between [2] and [3]. Now it is easy to shew that $\boldsymbol{A}$ does lie between these limits. For the quantities [1], [2], [3], [4], are the values of the expressions

$$
\frac{1}{2}(\boldsymbol{A}+\boldsymbol{B}) \pm \sqrt{ }\left(\frac{1}{2}(\boldsymbol{A}-\boldsymbol{B})^{2}+\boldsymbol{H}^{2}\right), \quad \frac{1}{2}(\boldsymbol{A}+\boldsymbol{C}) \pm \sqrt{ }\left(\frac{1}{2}(\boldsymbol{A}-\boldsymbol{C})^{2}+\boldsymbol{G}^{2}\right),
$$

Crelle's Journal f. d. M. Bd. XLVII. Heft 3. 
which may obviously be written in the form

$$
\boldsymbol{A} \pm \boldsymbol{C}, \quad \boldsymbol{A}+\boldsymbol{C}_{1}
$$

where $C$ and $C_{1}$ are positive. And it is plain that either $A \pm C$ are the values of [1] and [4] and $A \pm C_{1}$ of [2] and [3]; or vice versa. In either case $A$ lies between [2] and [3], and therefore also so does the fourth root of $\boldsymbol{S}$. And since for values of $\theta$ between [2] and [3], $G$ and $H$ are both positive, the root in question gives real values of the direction-cosines.

These conclusions may be recapitulated as follows.

Let $\theta^{\prime}, \theta^{\prime \prime}$ be two roots of that one of the three equations

$$
\mathbf{F}=\mathbf{0}, \quad \mathbf{G}=\mathbf{0}, \quad \mathrm{H}=\mathbf{0},
$$

whose roots are closet together, and $\theta_{1}, \theta_{u}$, the roots of that one that are farthest a part. Then of the three roots of the cubic [4], two lie respectively without the limits $\theta_{1}, \theta_{1}$, and the third within the limits $\theta^{\prime}, \theta^{\prime \prime}$; and this third root gives an unique system of real values to the direction-cosines $l, m, n$; $l^{\prime}, \boldsymbol{m}^{\prime}, \boldsymbol{n}^{\prime}$; thus determining an unique pair of real cyclic planes.

It would be easy to examine the peculiarities of the cases in which the cubic has equal or evanescent roots. But I will not pursue the subject farther.

It should be added that the limits of the roots of the cubic [4] were originally tracted by M. Cauchy (see Exercices de Math. 1828. p. 9.), but in a manner less simple, as it appears to me, than the above.

Oxford, March 24. 1853.

\section{W. F. Donkin.}

.. . Your method in applicable to the case of four variables, and when so applied, gives rise to some interesting geometrical results. Let

(1) $\boldsymbol{U}=\boldsymbol{A} \boldsymbol{x}^{2}+\boldsymbol{B} \boldsymbol{y}^{2}+\boldsymbol{C} \boldsymbol{z}^{2}+\boldsymbol{E} \boldsymbol{w}^{2}$

$$
+2(\boldsymbol{H} y z+G z x+H x y+L w x+M w y+N w z)=0 \text {, }
$$

then, if $U$ can be put in the form

(2) $\theta\left(x^{2}+y^{2}+z^{2}+w^{2}\right)+(l x+m y+w z+k w)\left(l^{\prime} x+m^{\prime} y+n^{\prime} z+k^{\prime} w\right)=0$ we have, writing

$$
\begin{aligned}
& \left\{\begin{array}{l}
x^{2}+y^{2}+z^{2}+w^{2}=\rho^{2}, \\
l x+m y+n z+k w=u, \\
l^{\prime} x+m^{\prime} y+n^{\prime} z+k^{\prime} w=v:
\end{array}\right. \\
& \text { (4.) } \quad U-\theta \rho^{2}=u v .
\end{aligned}
$$


Hence, comparing coefficients:

$$
\begin{gathered}
\text { (5) } \frac{l l^{\prime}}{A-\theta}=\frac{m m^{\prime}}{B-\theta}=\frac{n n^{\prime}}{C-\theta}=\frac{l k k^{\prime}}{E-\theta} \\
=\frac{m n^{\prime}+m^{\prime} n}{2 F}=\frac{n l^{\prime}+n^{\prime} l}{2 G}=\frac{l m^{\prime}+l^{\prime} m}{2 H}=\frac{l k^{\prime}+l^{\prime} k}{2 L}=\frac{m k^{\prime}+m^{\prime} k}{2 M}=\frac{n k^{\prime}+n^{\prime} k}{2 N^{\prime}}
\end{gathered}
$$

also, writing

$$
\left\{\begin{array}{l}
\boldsymbol{A}-\boldsymbol{\theta}=\boldsymbol{A}^{\prime}, \quad \boldsymbol{B}-\boldsymbol{\theta}=\boldsymbol{B}^{\prime}, \quad \boldsymbol{C}-\boldsymbol{\theta}=\boldsymbol{C}^{\prime}, \quad \boldsymbol{E}-\boldsymbol{\theta}=\boldsymbol{E}^{\prime} \\
\boldsymbol{H}^{2}-\boldsymbol{B}^{\prime} \boldsymbol{C}^{\prime}=\mathrm{F}, \quad \boldsymbol{G}^{2}-\boldsymbol{C}^{\prime} \boldsymbol{A}^{\prime}=\mathrm{G}, \quad \boldsymbol{H}^{2}-\boldsymbol{A}^{\prime} \boldsymbol{B}^{\prime}=\mathrm{H} \\
\boldsymbol{L}^{2}-\boldsymbol{A}^{\prime} \boldsymbol{E}^{\prime}=\mathrm{L}, \quad \boldsymbol{M}^{2}-\boldsymbol{B}^{\prime} \boldsymbol{E}^{\prime}=\mathrm{M}, \quad \boldsymbol{N}^{2}-\boldsymbol{C}^{\prime} \boldsymbol{E}^{\prime}=\mathrm{N}
\end{array}\right.
$$

each of these ratios is equal to

$$
\frac{m n^{\prime}-m^{\prime} n}{2 \sqrt{ } \mathrm{F}}=\frac{n l^{\prime}-n^{\prime} l}{2 \sqrt{\mathrm{G}}}=\frac{l m^{\prime}-l^{\prime} m}{2 \sqrt{\mathrm{H}}}=\frac{l k^{\prime}-l^{\prime} k}{2 \sqrt{\mathrm{L}}}=\frac{m k^{\prime}-m^{\prime} k}{2 \sqrt{\mathrm{M}}}=\frac{n k^{\prime}-n^{\prime} k}{2 \sqrt{\mathrm{N}}},
$$

from which, combined with (5), we derive the following systems:

(8)

$$
\begin{aligned}
& \mid \frac{m}{n}=\frac{F+\sqrt{ } \mathrm{F}}{C^{\prime}}=\frac{B^{\prime}}{F-\sqrt{ } \mathbf{F}}=\frac{H-\sqrt{ } \mathbf{H}}{G+\sqrt{G}}=\frac{M+\sqrt{ } M}{N+\sqrt{ } \mathbf{N}}, \\
& \frac{n}{l}=\frac{G+\sqrt{ } \mathrm{G}}{A^{\prime}}=\frac{C^{\prime}}{G-\sqrt{G}}=\frac{F-\sqrt{ } \mathbf{F}}{H+\sqrt{H}}=\frac{N+\sqrt{ } \mathbf{N}}{L+\sqrt{ } \mathbf{L}} . \\
& \left\{\frac{l}{m}=\frac{H+\sqrt{ } \mathrm{H}}{B^{\prime}}=\frac{A^{\prime}}{H-\sqrt{ } \mathrm{H}}=\frac{G-\sqrt{ } \mathrm{G}}{F+\sqrt{ } \mathbf{F}}=\frac{L+\sqrt{ } \mathbf{L}}{M+\sqrt{ } \mathbf{M}} .\right. \\
& \frac{l}{k}=\frac{L+\sqrt{ } \mathbf{L}}{E^{\prime}}=\frac{A^{\prime}}{L-\sqrt{ } \mathbf{L}}=\frac{H+\sqrt{ } \mathbf{H}}{M-\sqrt{M}}=\frac{G-\sqrt{G}}{N+\sqrt{ } \mathbf{N}}, \\
& \frac{m}{\boldsymbol{k}}=\frac{\boldsymbol{M}+\sqrt{ } \mathbf{M}}{\boldsymbol{E}^{\prime}}=\frac{\boldsymbol{B}^{\prime}}{\boldsymbol{M}-\sqrt{\mathbf{M}}}=\frac{\boldsymbol{F}+\sqrt{\mathbf{F}}}{\boldsymbol{N}-\sqrt{\mathbf{N}}}=\frac{\boldsymbol{H}-\sqrt{ } \mathbf{H}}{\boldsymbol{L}+\sqrt{ } \mathbf{L}}, \\
& \frac{n}{k}=\frac{N+\sqrt{ } \mathbf{N}}{E^{\prime}}=\frac{C^{\prime}}{N-\sqrt{ } \mathbf{N}}=\frac{G+\sqrt{G}}{L-\sqrt{ } \mathbf{L}}=\frac{F-\sqrt{ } \mathbf{F}}{M+\sqrt{M}},
\end{aligned}
$$

with a corresponding set of values for $l^{\prime}: m^{\prime}: n^{\prime}: k^{\prime}$, obtained. from (8) by writing $F \mp \sqrt{ } F$ etc. instead of $F \pm \sqrt{ } F$ etc. troughout. From these may be obtained the equations of condition:

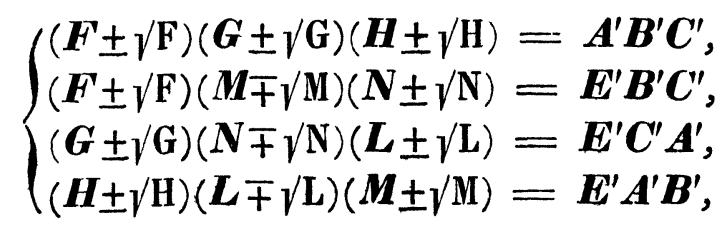

each of which, although apparently two distinct equations, is equivalent to only one, on account of the identities

$$
\left\{\begin{array}{cc}
\left(\boldsymbol{F}^{2}-\mathbf{F}\right)\left(\boldsymbol{G}^{2}-\mathbf{G}\right)\left(\boldsymbol{H}^{2}-\mathbf{H}\right)=\boldsymbol{A}^{2} \boldsymbol{B}^{\prime 2} \boldsymbol{C}^{\prime 2}, \\
.
\end{array}\right.
$$


and the system is finally equivalent to only three independent conditions on account of the identity

$$
\text { (11) }\left(L^{2}-\mathbf{L}\right)\left(M^{2}-\mathrm{M}\right)\left(\boldsymbol{N}^{2}-\mathbf{N}\right)=\boldsymbol{E}^{\prime 3} \boldsymbol{A}^{\prime} \boldsymbol{B}^{\prime} \boldsymbol{C}^{\prime}
$$

It may be noticed in passing that two of the conditions (9) may be thrown into the form

$$
\left\{\begin{array}{l}
\frac{M+\sqrt{ } M}{M-\sqrt{M}}: \frac{N+\sqrt{ }}{N-\sqrt{N}}=\frac{F+\sqrt{F}}{F-\sqrt{ }} \\
\frac{N+\sqrt{ }}{N-\sqrt{ }}: \frac{L+\sqrt{ }}{L-\sqrt{L}}=\frac{G+\sqrt{G}}{G-\sqrt{G}} \\
\frac{L+\sqrt{L}}{L-\sqrt{ } \mathbf{L}}: \frac{M+\sqrt{M}}{M-\sqrt{M}}=\frac{H+\sqrt{H}}{H-\sqrt{ }}
\end{array}\right.
$$

to which others might be added.

The conditions (9) may likewise be put in a form better known, as follows. Developping the first pair of equations, and subtracting the results, we have

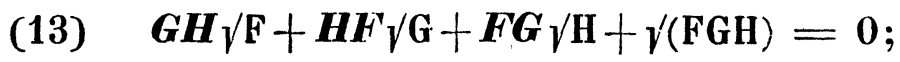

adding the results, we have

$$
\text { (14) } \quad \boldsymbol{F} \sqrt{ }(\mathrm{GH})+\boldsymbol{G} \sqrt{ }(\mathrm{HF})+\boldsymbol{H} \sqrt{ }(\mathrm{FG})+\boldsymbol{F} \boldsymbol{G H}-\boldsymbol{A}^{\prime} \boldsymbol{B}^{\prime} \boldsymbol{C}^{\prime}=0
$$

transponing the last termes of (13) and quadring:

$\mathbf{F} \boldsymbol{G}^{2} \boldsymbol{H}^{2}+\mathbf{G} \boldsymbol{H}^{2} \boldsymbol{F}^{2}+\mathbf{H} \boldsymbol{F}^{2} \boldsymbol{G}^{2}+2 \boldsymbol{F} \boldsymbol{G H}\left(\boldsymbol{F} \sqrt{ }(\mathbf{G H})+\boldsymbol{G}_{\sqrt{ }}(\mathbf{H F})+\boldsymbol{H}_{\sqrt{ }}(\mathrm{FG})\right)=\mathrm{FGH}$; substituting from (14), and dividing throughout by $\boldsymbol{A}^{\prime} \boldsymbol{B}^{\prime} \boldsymbol{C}^{\prime}$, there finally results:

$$
\boldsymbol{A}^{\prime} \boldsymbol{B}^{\prime} \boldsymbol{C}^{\prime}-\boldsymbol{A}^{\prime} \boldsymbol{F}^{2}-\boldsymbol{B}^{\prime} \boldsymbol{G}^{2}-\boldsymbol{C}^{\prime} \boldsymbol{H}^{2}+2 \boldsymbol{F} \boldsymbol{G H}=0 .
$$

By means of this reduction the system (9) admits of being expressed in a brief manner, as follows: Let

then (9) take the form

$$
\left\{\begin{aligned}
\Omega= & \boldsymbol{A}^{\prime} \boldsymbol{H} \boldsymbol{G} L \\
& \boldsymbol{H} \boldsymbol{B}^{\prime} \boldsymbol{F} \boldsymbol{M} \\
& \boldsymbol{G} \boldsymbol{F} \boldsymbol{C}^{\prime} \boldsymbol{N} \\
& \boldsymbol{L} M \boldsymbol{N} \boldsymbol{E}^{\prime},
\end{aligned}\right.
$$

$$
\text { (17) } \frac{d \Omega}{d A^{\prime}}=0, \frac{d \Omega}{d B^{\prime}}=0, \frac{d \Omega}{d C^{\prime}}=0, \frac{d \Omega}{d E^{\prime}}=0 .
$$

Writing, for convenience,

$$
\left\{\begin{aligned}
-(\boldsymbol{A}+\boldsymbol{B}+\boldsymbol{C}) & =(\boldsymbol{A}, \boldsymbol{B}, \boldsymbol{C})_{1} \\
-(\boldsymbol{B C}+\boldsymbol{C A}+\boldsymbol{A B})+\boldsymbol{F}^{2}+\boldsymbol{G}^{2}+\boldsymbol{H}^{2} & =(\boldsymbol{A}, \boldsymbol{B}, \boldsymbol{C})_{2} \\
\boldsymbol{A} \boldsymbol{F}^{2}+\boldsymbol{B} \boldsymbol{G}^{2}+\boldsymbol{C H}-\boldsymbol{A B C}-2 \boldsymbol{F} \boldsymbol{H} \boldsymbol{H} & =(\boldsymbol{A B C})_{3}
\end{aligned}\right.
$$


the four conditions (17) may be written:

$$
\left\{\begin{array}{l}
\theta^{3}+(\boldsymbol{A B C})_{1} \theta^{2}+(\boldsymbol{A B C})_{2} \theta+(\boldsymbol{A B C})_{3}=0, \\
\theta^{3}+(\boldsymbol{B C E})_{1} \theta^{2}+(\boldsymbol{B C} \boldsymbol{C})_{2} \theta+(\boldsymbol{B C E})_{3}=0, \\
\theta^{3}+(\boldsymbol{C E} \boldsymbol{A})_{1} \theta^{2}+(\boldsymbol{C E A})_{2} \theta+(\boldsymbol{C E} \boldsymbol{A})_{3}=0, \\
\theta^{3}+(\boldsymbol{E} \boldsymbol{A B})_{1} \theta^{2}+(\boldsymbol{E} \boldsymbol{A B})_{2} \theta+(\boldsymbol{E} \boldsymbol{A} \boldsymbol{B})_{3}=\mathbf{0}
\end{array}\right.
$$

and the two conditions resulting from the elimination of $\theta$ from any three of these equations will be those to which the quantities $A, B, C, F, G, H, L, M, N$, must be subject, in order that the transformation from (1) to (2) may be possible. But it does not seem worth, while to proceed to the development of the results, which would be a work of great labour.

It remains to determine the geometrical results, ensuing upon the transformation in question; the principal peculiarities of which depend on the value of the quantity $w$.

1. Let $w=0$; then the problem reduces itself to that considered in the latter part of your letter.

2. Let $w$ be a real constant; then the surface will be cut circularly by either of the planes

$$
\begin{aligned}
& l x+m y+n z+k w=\widetilde{\omega} \\
& l^{\prime} x+m^{\prime} y+n^{\prime} z+k^{\prime} w=\widetilde{\omega}^{\prime}
\end{aligned}
$$

(or, as they may be termed, the planes $\widetilde{\omega}, \widetilde{\omega}^{\prime}$ ). It may also be noticed that $\widetilde{\omega}, \widetilde{\omega}^{\prime}$ and $\widetilde{\omega}$ vanish together.

3. Let $w$ be an imaginary constant; then the surface is cut circularly by either of the imaginary planes $\widetilde{\omega}=0$, or $\widetilde{\omega}^{\prime}=0$, and also by the real hyperboloïd

$$
(l x+m y+n z)\left(l^{\prime} x+m^{\prime} y+n^{\prime} z\right)=k^{2} w^{2} .
$$

4. Let $w$ be a homogeneous linear function of $x, y, z ;$ e. g. let

$$
w=\lambda x+\mu y+\nu w
$$

then if the surface be cut by either of the planes

$$
\begin{aligned}
& u+k w=0, \\
& v+k w=0,
\end{aligned}
$$

i. e. by a plane making angles with the planes $u$ and $u$, or $v$ and $w$, the ratio of whose series is $=-k$; it will be cut also in its intersection with the surface

$$
x^{2}+y^{2}+z^{2}+(\lambda x+\mu y+\nu z)^{2}=0,
$$


and if moreover

$$
w_{1}=w \sqrt{ }-, \quad \lambda_{1}=\lambda \sqrt{ }-, \quad \mu_{1}=\mu \sqrt{ }-, \quad \nu_{1}=v-
$$

the two surfaces

$$
\begin{gathered}
\boldsymbol{w} v+k^{2} w_{1}^{2}=0 \\
x^{2}+y^{2}+z^{2}-\left(\lambda_{1} x+\mu_{1} y+v_{1} z\right)^{2}=0
\end{gathered}
$$

will both intersect (1) in the same curve. This latter surface has a double contact with the sphere

$$
x^{2}+y^{2}+z^{2}=\text { const. }
$$

and is cut circularly by the plane

$$
w_{1}=0
$$

a plane which cuts the former surface in the straight line

$$
\boldsymbol{u}=\mathbf{0}, \quad v=0 \text {. }
$$

It may further be remarked that the plane $w_{1}=0$ cuts the original surface in the same curve as does the cone

$$
\theta \boldsymbol{r}^{2}+\boldsymbol{u v}=\mathbf{0}
$$

whose cyclic planes are $u=0, v=0$.

London, March 26. 1853.

W. Spottiswoode. 